\title{
An artificial neural network-based diagnostic methodology for gas turbine path analysis-part II: case study
}

\author{
Roberto Capata ${ }^{1}$ \\ ${ }^{1}$ Department of Mechanical and Aerospace Engineering, University of Roma "Sapienza", Rome, Italy
}

Received: 21 September 2015/Revised: 9 September 2016/Accepted: 12 September 2016/Published online: 1 October 2016

(c) Joint Center on Global Change and Earth System Science of the University of Maryland and Beijing Normal University and Springer-Verlag Berlin Heidelberg 2016
Abstract The reliability of gas path components (com- pressor, burners and turbines) of a gas turbine is generally high, when compared with those of other systems. How- ever, in case of forced stops, downtime is usually high, with a relatively low availability. The purpose of condi- tions monitoring and faults diagnostics is to detect, isolate and evaluate (i.e., to estimate quantitatively the extent) defects within a system. One effective technique could provide a significant improvement in economic perfor- mance, reduce operating costs and maintenance, increase the availability and improve the level of safety achieved. However, conventional analytical techniques such as gas path analysis and its variants are limited in their engine diagnostic, due to several reasons, including their inability to effectively operate in the presence of noise measures, to distinguish anomalies of component from a failure sensor, to preserve the linearity in the relations between parameters of gas turbines and to manage the sensors range to achieve accurate diagnosis. In this paper, the approach of a diag- nostic scenario to detect faults in the gas path of a gas turbine has been presented. The model provides a large- scale integration of artificial neural networks designed to detect, isolate and evaluate failures during the operating conditions. The engine measurements are considered as input for the model, such as the speed, pressure, tempera- ture and fuel flow rate. The output supplies any changes in the sensor or in the efficiency levels and flow rate, in the event of fault components. The diagnostic method has the ability to evaluate both anomalies of multiple components or multiple sensors, within the range of operating points. In
Roberto Capata
roberto.capata@uniroma1.it
the case of components failures, the system provides diagnostic changes in efficiency and flow rate, which can be interpreted to determine the nature of the physical problem. The technique has been applied in different operating conditions by comparing the results obtained with the solutions provided by linear and nonlinear analysis.
Keywords Gas turbine diagnostic - Gas path analysis . Neural network

$\begin{array}{ll}\text { Abbreviations } & \\ \text { ANN } & \text { Artificial neural network } \\ \text { APPROX } & \text { Approximation network } \\ \text { ATT } & \text { Average training time } \\ \text { AUTOASSOC } & \text { Auto-associative network } \\ \text { C } & \text { Compressor/category } \\ \text { CBM } & \text { Component base monitoring } \\ \text { CC } & \text { Combustion chamber } \\ \text { CF } & \text { Component fault } \\ \text { CoC } & \text { Coefficient of correlation } \\ \text { COV } & \text { Covariance } \\ \text { DCF } & \text { Double-component failure } \\ \text { DSF } & \text { Double sensor failure } \\ \text { F } & \text { Fault, failure } \\ \text { G } & \text { Generator } \\ \text { GPA } & \text { Gas path analysis } \\ \text { GPF } & \text { Gas path failure } \\ \text { GPFD } & \text { Gas path fault diagnosis } \\ \text { GT } & \text { Gas turbine } \\ \text { I } & \text { Intermediate compressor } \\ \text { MBD } & \text { Model-based diagnostic } \\ \text { MCF } & \text { Multiple-component fault } \\ \end{array}$




$\begin{array}{ll}m & \text { Component mass flow rate } \\ N & \text { Number of tests (Sect. 3) } \\ \text { NF } & \text { No fault } \\ P & \text { Shaft power } \\ p & \text { Pressure } \\ \text { SCF } & \text { Single-component failure } \\ \text { SF } & \text { Sensor fault } \\ \text { SP } & \text { Shaft power } \\ \text { SSF } & \text { Single-sensor failure } \\ T & \text { Temperature/turbine } \\ \text { TBO } & \text { Time between overhaul } \\ \text { TCF } & \text { Triple-component FAULT } \\ X, Z, z & \text { Input parameters }\end{array}$

\section{Greek symbol}

$\eta \quad$ Efficiency

$\sigma \quad$ Standard deviation

$\Delta$ Difference

\section{Subscripts}

a Ambient

bki Corrected reference for sensor " $\mathrm{i}$ " on " $\mathrm{k}$ " model

c Compressor

cki Network prevision for sensor "i" on " $k$ " model

i Sensor

ki K model sensor

$\mathrm{T}$ Turbine

\section{Introduction}

The turbine plays an increasingly important role in the energy generation and distribution. This aspect leads to improve economic performance, by improving the efficiency of components, extending the periods between maintenance (TBOS) and increasing the overall performance. The cost per kilowatt value produced by a GT $(€ /$ $\mathrm{kW}$ ) is one of the most aspects in the plant management and includes all necessary costs to maintain the turbine operational. The efficiency variation should be considered in the calculation of the plant life cycle. The availability of gas path components might be relatively low because high downtime is normally associated with forced stops. A possible solution to increase the availability is to improve the maintenance operations, basing on monitoring conditions (CBM). Before the advent of the GPA (gas path analysis) tools, the gas path failures have been diagnosed mainly by means of quality tools, by matching symptoms to pre-established patterns (to identify the tendency to the variation in the engine measurements and then isolate a failing module or component). This approach does not consider that the apparent fault could be due to measurement noise. The solutions cannot be provided by qualitative approaches, both conventional and evolutionary. GPA and its variants, as well as some artificial intelligence techniques, are able to provide quantitative results for the breakdowns in the gas path, as shown in Table 1.

1. The used ANN methodology

In the first part, it was exposed as an ANN model, which can be used to perform four basic functions in a gas turbine plant: to generate performance parameters, estimate the plant/process operating conditions, estimate the sensor data and, finally, validate sensors data using the separation of incorrect/noise measurements from good ones. At the end of the first phase, correction factors for the proposal ANN method have been calculated. The next step will be to apply this methodology and calculated correction factors in gas turbine plant, to evaluate the choices and verify the validity of correction introduced, with the aim to separate the noise/incorrect measures from the correct ones.

2. The gas path fault diagnosis model evaluation

The diagnostic approach is presented, with case studies based on typical engine configurations (single shaft), in stationary conditions. The neural network architectures developed for these modified engine configurations are not unique, except for the engine name plate data (Boyce 1984), used for the analysis. They are generic for GT engines with similar configurations. For complex engine configurations such as those incorporating intercooler, an adjustment would be required on diagnostic structure presented (this situation has been not considered here). The first problem is to evaluate the highly nonlinear relationship between performance parameters and the parameters measured (Hajagos and Bérubé 2001; Centeno et al.; Agrawal et al. 1978). It can be noticed that the aim of research is to separate the sensor faults by the system faults. In fact, it is possible that degradation of performance parameters, considered insignificant in terms of operability, can cause large changes in sensor measurements. Similarly, by a significant degradation in a single performance parameter, it might be similar than the standard noise level.

The procedure is as follows. The system has been simulated using numerical codes [developed in the Italian Energy and Environment National Agency-ENEA (ENEA Personal Communication 2016)] and then experimentally tested in their laboratories, where the GT device was available. The data were collected and compared with the available ones. This first step has allowed to establish the operational steps, identifying the contribution in the measure of the "white noise".

Considering these issues, a preliminary analysis is needed to determine what level of performance parameters can be determined from measurements in noisy conditions. Besides, it must simultaneously ensure that such 
Table 1 Gas turbine fails and failures

\begin{tabular}{lll}
\hline Compressor & Turbine & Combustor \\
\hline Fouling & Fouling & Corrosion \\
Blade fatigue & Corrosion & Cracking \\
Blade pitting, blade corrosion & Hot corrosion, sulphidation & Nozzle imbalance \\
surge & FOD/DOD & Fuel quality \\
Corrosion, erosion & Excessive back pressure & Vibration and pulsation \\
LCF/HCF & Creep & Clogging \\
FOD & Nozzle bowing & Leakages \\
& Bearings distress & Fretting corrosion \\
& HCF/LCF & \\
\hline Filter & Blade coating & \\
\hline Fouling & & Mechanical problems \\
Clogging & & Bearings problems \\
Icing & & Critical speeds \\
Airflow distortion & & Unbalance \\
Humidity effects & & Looseness and misalignment \\
Loss of air tightness & & Foundations \\
\hline & & Rotor bows \\
\hline
\end{tabular}

deviations, in measured parameters, are not limited to one or two sensors. This aspect, in fact, would prevent proper distinction of sensors failures and components failures.

Besides, it has made possible to validate the simulation code.

Later changes in various parameters have been introduced. Once again, the data have been compared and inserted into the database of the algorithm of diagnosis. This analysis has provided the minimum level of failure to be implemented on engine models, to generate degradation data.

Finally, changes in functional parameters such as to induce or generate a fault have been introduced. The value entered was taken from experimental values and from the available literature.

The failure analysis, for both component and sensor, requires a baseline, from which the deviations can be obtained, in reference to an established operating point.

It is important to remember that the strengths of neural networks are the ability to reliably work even with corrupted data/noisy. To construct a neural network with due robustness, the data generated are contaminated with noise, before the training and testing of each network start. The system should be able to detect both the individual and multiple components breakdowns. These considerations required a very large database. This database consists of several pairs of performance arrays, called a pattern. This creates a huge library of failures, from which they designed the patterns of training and testing. Thanks to our adopted approach, the initial failure for the first network classifier is generic, but subsequent networks become more specific (realized to isolate or evaluate case faults). The philosophy of the proposed approach is such that the presence of a failure is first ensured (detected), isolated its origin and evaluated its impact.

\subsection{The preliminary GPFD application: single shaft}

Following the several researchers suggestion and proposals (Angelakis et al. 2001; Brotherton et al. 2000; DePold and Gass 1999), a genetic algorithm [developed in collaboration with ENEA (ENEA Personal Communication 2016)] composed by a thirteen neural network architectures, including 5 classification networks, 1 auto-associative network, and 4 approximation networks, has been considered (Fig. 1).

Engine data are introduced into the network as deviations from a reference value. The first classification CATEGORY 1 (from now defined as $\mathrm{C} 1$ ) network evaluates and classifies them as not failure (NF) or failure (F). If the model is not defective, the process terminates. If a failure occurs, the data are transmitted to the second classification network, $\mathrm{C} 2$, which determines whether the fault is due to faulty sensor error or component failure. If the fault is identified as a sensor error, an auto-associative network (from now A1) is called to determine the magnitude of the failure (s) in the polarized sensor. If the model is classified as a component failure from $\mathrm{C} 2$, a third network of classification, $\mathrm{C} 3$, is called to determine whether the model is faulty as a result of a single-component failure 


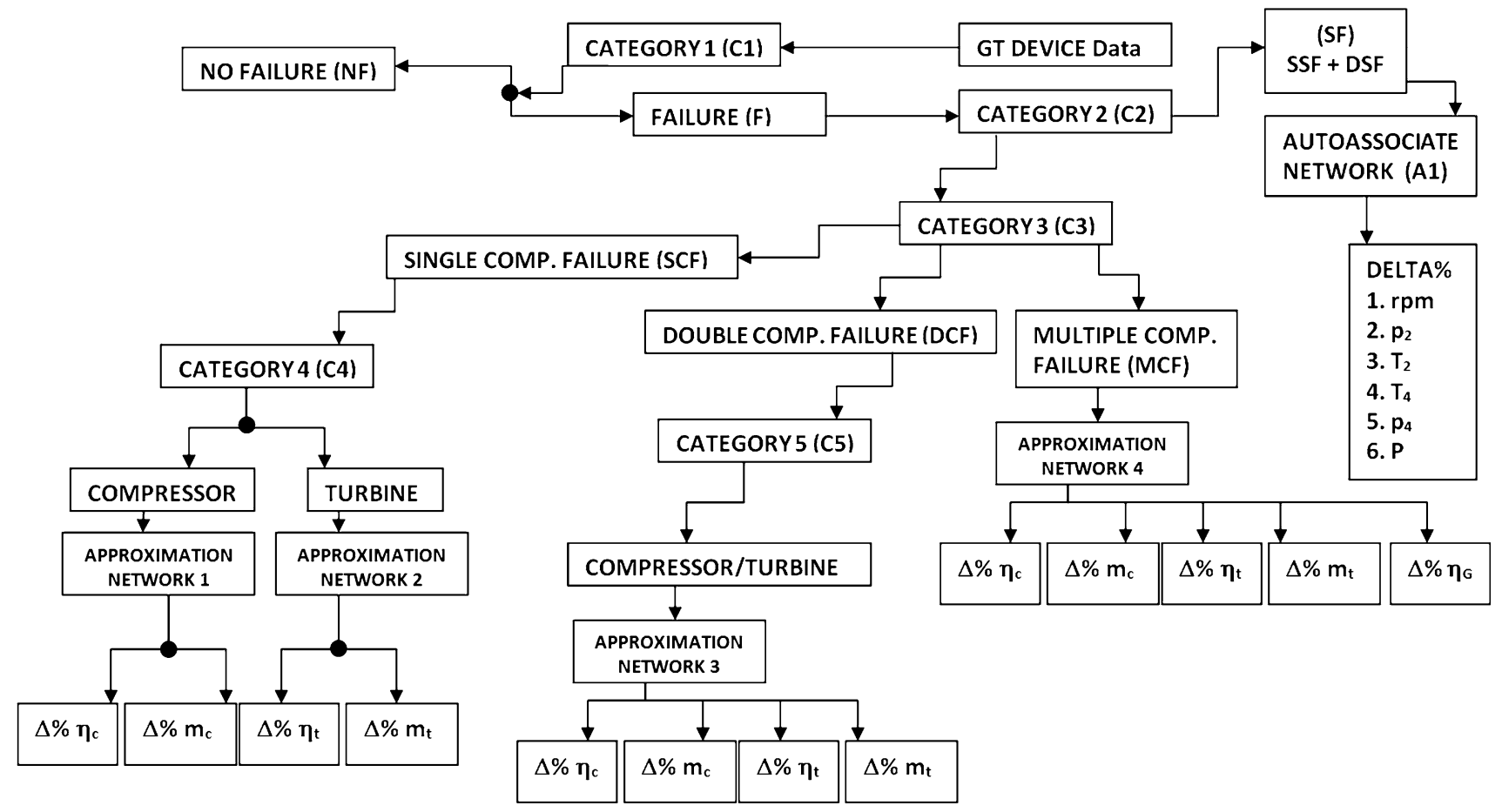

Fig. 1 A possible GPFD-ANN module for single-shaft gas turbine device (Aker and Saravanamuttoo 1989)

(SCF), a double-component failure (DCF) or a multicomponent failure (MCF) (Fantoni and Mazzola 1996). If the failure reason is identified as a SCF by the $\mathrm{C} 3$ network, the template is forwarded to a fourth classification network, $\mathrm{C} 4$, to isolate the single-component failure, which could be both the compressor and the turbine. When the failing component was isolated, the pattern is transferred to a network of approximation, which determines the extent of the component deterioration. This will determine abnormalities affecting two concurrent components.

\subsection{Sensors fault detection, isolation and quantification}

Referring to the failure level, the sensors failure can be classified into two categories (Tamuno-Ojuemi 2003):

1. Warning Failure: large but easy to detect.

2. Danger Failure: difficult to locate and, if not compensated, potentially catastrophic.

This network requires that the number of "neurons" in bottleneck is greater or equal than the number of main components, needed to reconstruct the exit, in case of sensor failure (s) or noisy inputs. In addition, the size of the input and output models can generate a particular output pattern. This makes the neural network separate from hetero-associative networks, where different input patterns can be mapped with various output collections, with input and output dimensions not necessarily identical. The training was created in four target groups, which are:

1. No noise or disturbance;

2. Zero-mean Gaussian noise;

3. Single-sensor failure that produces fault classes equal to the number of sensors;

4. Dual-sensor failure: Using the combination relationship in Eq. (1):

Fault category $=\mathrm{nCr}_{\text {bias }}=\left(\begin{array}{c}n \\ r_{\text {bias }}\end{array}\right)=\frac{n !}{r_{\text {bias }} !\left(n-r_{\text {bias }}\right) !}$

15 sensors failure classes for a stationary single-shaft GT were obtained, while 28 sensor failure classes for the GT engine with 8 (eight) measurements were obtained.

The auto-associative networks for single-shaft GT and single turbo engine propeller were then trained to isolate and quantify the errors of single and double sensors and noise filtering. The implemented sensor error varies from the low value (two times the noise level) up to the limit $(+10 \%$ of deviation). All network data have been processed before and after its generation to realize an accurate data exit. Equations (2-5) were used (Tamuno-Ojuemi 2003; Anderson and McNeill 1992; Cheng and Titterington 1994). 
$I_{i}=\left(Z_{\mathrm{ki}}-Z_{\mathrm{bki}}\right) / Z_{\mathrm{bki}} \cdot 100$

$O_{i}=\left(Z_{\mathrm{cki}}-Z_{\mathrm{bki}}\right) / Z_{\mathrm{bki}} \cdot 100$

$\% \Delta$ sensor fault or noise $=I_{i}-O_{i}$

$Z_{\mathrm{cki}}=1 / 100 \cdot\left(Z_{\mathrm{bki}} \cdot O_{i} \cdot Z_{\mathrm{bki}}\right)$

Equations (2) and (3) were used to generate the input and output of the network training and test the network itself (Bakal et al. 1995; Barlett and Uhrig 1992). Equation (4) was used to determine the level failures or noise, related to any input. This is the result of fault isolation by self-associative networks and of the quantification process. Equation (5) was applied to reconstruct the approximate output sensor, once noise is filtered. The network output, during training, was limited within the correct/clean range (i.e., without noise or uncertainty). For the components fault diagnosis, the data have been generated in the failure classes for the three configurations given in Table 2.

Process flow diagram is reported in Fig. 2.

\section{Results analysis}

\subsection{Network classification}

The diagnostic model, developed in the previous section, has undergone a series of tests, carried out with a software developed by ENEA, UDR1 and the manufacturer (for these reasons, some parts cannot be more detailed, due to a non-disclosure agreement). The input and output contribution can be determined by applying the concept of casual importance (Aker and Saravanamuttoo 1989), or using input removal techniques. So, each node output is generated by different network inputs. Actual GPA can handle the tasks, with a reasonable accuracy. In this way, the explanation of the results of classification would be based on those neurons that are exciting and those are not energized.

The discussion of results using the approximation would be based on statistical comparison with expected or reference values. $\mathrm{C} 1$, which indicates that measurements on an engine without defects, has to be properly identified (Tamuno-Ojuemi 2003). If a fault that does not exist, the engine must continue to operate, and it is identified as operational. This level of accuracy was possible by

Table 2 Breakdown classes of the single-shaft GT

\begin{tabular}{ll}
\hline Fault class & Component(s) \\
\hline 1 & $\mathrm{C}$ \\
2 & $\mathrm{~T}$ \\
3 & $\mathrm{C} / \mathrm{T}$ \\
4 & $\mathrm{G}$ \\
\hline
\end{tabular}

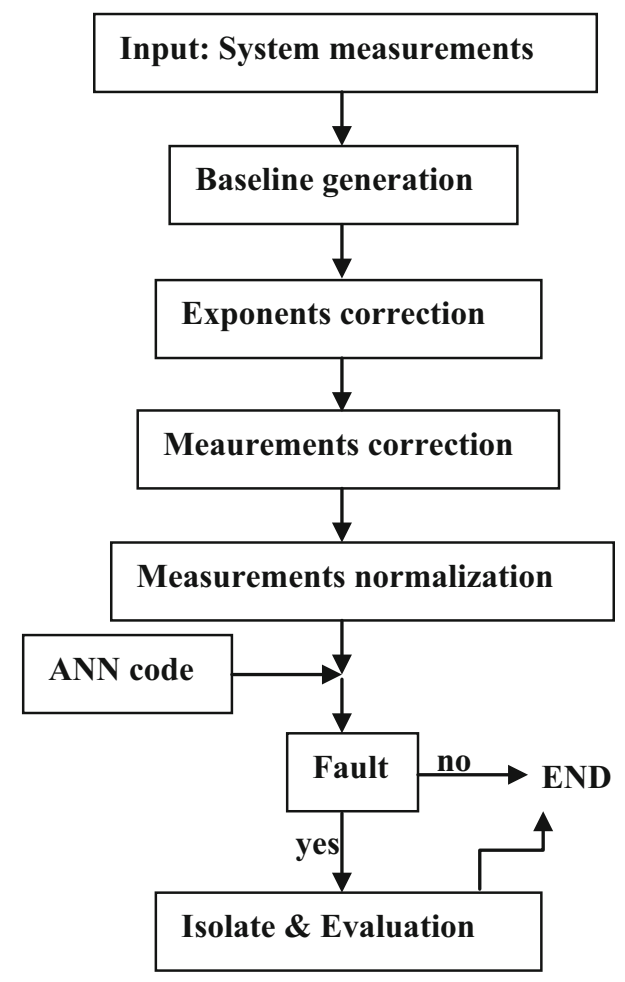

Fig. 2 Process flowchart

simulating thousand of patterns. In fact, operational conditions, as well as failures and sensor uncertainties, are introduced in the code to train the network. As a matter of fact, the $\mathrm{C} 1$ has identified all models of NF and identified the $99.9 \%$ of breakdowns, during our simulations. Among the 12 wrongly classified models, 1 (one) belonged to the class of component failure with very minor defect, while the remaining 11 belonged to the category of the sensor failure with the lower value. This low level of failure generates charts similar to a NF condition, which leads to a misclassification. However, the results of the $\mathrm{C} 1$ indicate that the ability to isolate a faulty measurement from a no fault can be adequately handled by the program trained network.

The network $\mathrm{C} 2$ is designed to distinguish a sensor failure from a component failure. It is important to remember that the actual diagnostic programs are unable to distinguish among a component (s) failure and a faulty sensor (s). In fact, these sensor faults (SFs) are interpreted as a failure of engine components, with long unprofitable downtime. Acting as previously described for $\mathrm{C} 1$, the results show that a well-trained $\mathrm{C} 2$ can reach over $99 \%$ accuracy of classification for this task. Models with low failures in multiple sensors have graphics similar to those obtained from low-fault components. Consequently, they generate regions, where both classes are equally probable. The natural consequence of this is a wrong classification, 
Table 3 Classification of network results (Aker and Saravanamuttoo 1989)

\begin{tabular}{|c|c|c|c|}
\hline \multirow[t]{2}{*}{ C 1} & No failure (NF) & Failure $(\mathrm{F})$ & \\
\hline & 1200 & 9896 & \\
\hline$\% \mathrm{CCP}$ & 99.7 & 99.8 & \\
\hline \multirow[t]{2}{*}{ C 2} & Sensor failure (SF) & Component failure $(\mathrm{CF})$ & \\
\hline & 1800 & 9601 & \\
\hline$\% \mathrm{CCP}$ & 99.2 & 99.8 & \\
\hline \multirow[t]{2}{*}{ C 3} & $\mathrm{SCF}$ & DCF & $\mathrm{MCF}$ \\
\hline & 1300 & 4006 & 4396 \\
\hline$\% \mathrm{CCP}$ & 98.9 & 93.8 & 88.9 \\
\hline \multirow[t]{2}{*}{ C 4} & $\mathrm{C}$ & $\mathrm{T}$ & \\
\hline & 558 & 362 & \\
\hline$\% \mathrm{CCP}$ & 99.99 & 99.9 & \\
\hline \multirow[t]{2}{*}{ C 5} & $\mathrm{C} / \mathrm{T}$ & & \\
\hline & 1518 & & \\
\hline$\% \mathrm{CCP}$ & 98.5 & & \\
\hline
\end{tabular}

but the result indicates that this issue appears to be minimal (Table 3).

C3 is affected by the lower value of accuracy. This is due to the complexity of the task and due to the network size increase. A number of overlaps were observed among the three considered category but most especially between the double-component failure (DCF) and triple-component failure (TCF). In fact, considering a simulation composed by $1300 \mathrm{SCF}$ patterns, the $\mathrm{C} 3$ has been correctly classified the $98.9 \%$ of the results, while 10 patterns are classified as DCF and two (2) patterns as TCF. Analyzing the results, it has been noticed how some SCF patterns have characterized by a high failure level similar to those from DCF, where one component (compressor or turbine) has a high failure value and the other a lower one. Analogously, an high DCF can be interpreted as TCF, due to the similar signature (Dong et al. 1997; Barschdorff 1991). These findings may still be used. Empirical studies have shown that when an SCF is classified as a DCF, the error in the defective component is defined correctly, while the second component is assigned a value lower than that actually diagnosed. The reverse is also true, i.e., if a DCF is considered or construed as an SCF. Here, the component with the higher fault is isolated and the amount of fault quantified. If this fault is corrected and the measurements retaken and introduced into the network, the previously unidentified component would be isolated and its fault level assessed.

The classification accuracy obtained from $\mathrm{C} 4$ demonstrates the high level of distinction that exists between the three classes of failure. As a result, the training time is low, as the size of the network. $\mathrm{C} 4$ insulates the failure at the level of components, before evaluating the failing component.

\subsection{The approximation NETWORK}

A diagnostic code has to identify the failure component and estimate the error amount. The failures must be isolated to the component level(s), before making a failure assessment. So, to determine which component(s) is responsible for the decrease in performance is only one aspect of the problem. The other aspect is the estimate of the amount of failure. The definition of the error standard deviation $(\sigma)$ represents an indication of how far, in the error space, it is needed to study the failures that consider a given percentage of the test data (Féraud and Clérot 2002). For example, a standard error deviation $(1-\sigma)$ includes about $68 \%$ of test data $(2-\sigma)$ approximately $95 \%$, while $(3-\sigma)$ includes approximately $99 \%$. In this analysis, a $(2-\sigma)$-level error, to determine a sufficiently accurate interval with average errors about zero, has been used. To distinguish error values deriving from different levels of failure, the error equation, defined by Eq. (6), is introduced.

$E_{\text {rel }}=\left(\rho-\rho_{\text {actual }}\right) / \rho_{\text {actual }}$

The standard deviation is calculated with relation (7)

$\sigma=\sqrt{\frac{\sum_{i=1}^{N}\left(E_{\mathrm{rel}(1)}-\bar{E}_{\mathrm{rel}}\right)^{2}}{N-1}}$

where $N$ is the number of test models.

It follows, therefore, that the network should be estimated in the range defined in Eq. (7) for the $95 \%$ of the time.

$\rho=\rho_{\text {actual }} \pm 2 \sigma$

Another approach, in the analysis of approximation results, is the correlation coefficients $(\mathrm{CoC})$ method.

The $\mathrm{CoC}$ is a measure of how the correct trends, in expected value, are consistent with trends in actual/target values. The $\mathrm{CoC}$ in this case was calculated by the equation:

$$
\begin{aligned}
\mathrm{CoC} & =r=\frac{n \sum a t-\sum a \sum t}{\sqrt{\left[n \sum a^{2}-\left(\sum a\right)^{2}\right] \cdot\left[n \sum t^{2}-\left(\sum t\right)^{2}\right]}} \\
& =\frac{\operatorname{COV}(a, t)}{\sigma_{a} \cdot \sigma_{t}}
\end{aligned}
$$

where $\mathrm{a}$ is the prediction of a network performance parameter; $t$ is the target or actual value of the performance parameter; $\mathrm{n}$ is the number of data points; $\mathrm{COV}$ is the covariance between the network and reference output. 
If the relationship between an expected value and real value increases, also the $\mathrm{CoC}$ tends to 1 , while a "weakened relationship" tends to -1 for reversed trends. Correlation coefficients $(\mathrm{CoCs})$ are calculated for each performance parameter in various approximating networks. The flow rate of $\mathrm{CoC}$ indicates a much stronger relationship between the network and the standard values, compared to the efficiency CoC. This simply means that the networks better approximate the flow rate prediction than the efficiency increase. In terms of obtained absolute errors, by a direct comparison between the failure estimation and the exact fault levels, the sizes are equivalent. But, relatively to the estimated failures values, errors in flow rate are higher compared to those obtained from the efficiency estimation. In a compressor, the error range for the efficiency is between -3.5 and $-0.5 \%$, while for the flow rate is among -17.5 and $+3.5 \%$. In the turbine efficiency, changes are similar to the compressor ones, but the flow rate varies from -3.5 to $+10.5 \%$. These values were chosen to cover a wide range of possible faults. Percentages errors are calculated by Eq. (8).

\subsection{Sensor fault diagnosis}

The auto-associative networks (AUTOASSOC) are responsible for isolating the sensor failure and the estimation of the value. The number of diagnosed faulty sensors is inversely proportional to the number of neurons required in the bottleneck to reconstruct the output in case of sensor failure (s) or noisy inputs (Barschdorff 1991; Brownell 1992).

It is important to notice that the values, for the sensor not repeatability, have produced a noise level and added to the sensor readings. This has made possible to train networks and define a minimum value, for which a given reading sensor is identified as defective. This difference is needed to create a certain gap/difference between a fault and a class of non-defective sensor. Then, a white noise, with a normal distribution to all classes and test data, has been added. Failure values for all sensors implanted are twice the level of noise and about $20 \%$ of the same level. The outputs from the network during the training have been filtered using auto-associative neural network.

To train the network, changes onto the values of the sensors have been introduced. The errors introduced are related to the GT efficiency and the mass flow rate. These variations are about $5 \%$ (Aker and Saravanamuttoo 1989; Tamuno-Ojuemi 2003). These variations are completely random. Finally (choosing some patterns, in our case a dozen), it is possible to evaluate the error on the sensor reading and then verify whether the network is able to recognize it as a sensor defect and not the component. The

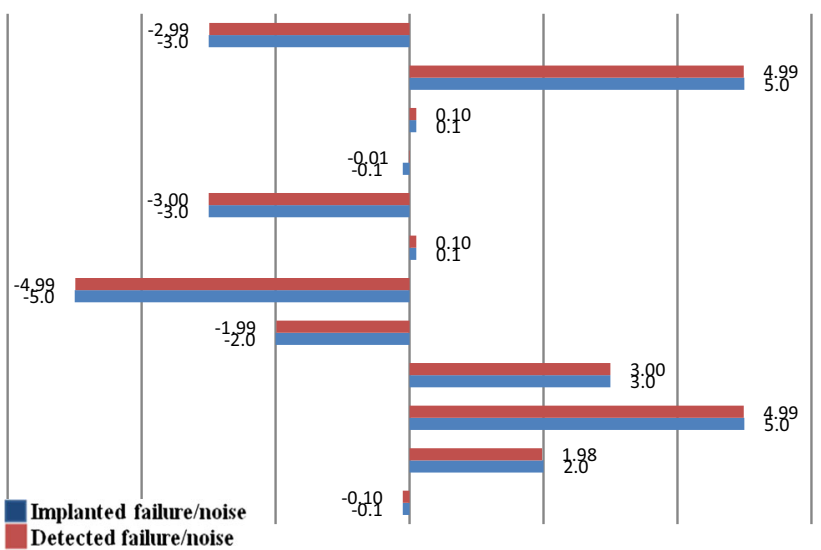

Fig. 3 Comparison between implanted and detected failures for a $\omega_{1}$ single sensor (Aker and Saravanamuttoo 1989)

results of this comparison are reported in Fig. 3, where the only $\omega_{1}$ sensor is shown.

\section{Conclusions and possible remarks}

The literature has indicated critical functionality, which must be considered, in the ANN application. The model studied in this work had similar thermodynamic features of the actual prototypes. The sensors have been chosen based on their quality, i.e., in relation to the fault diagnosed observability. A parameter-correction approach is useful for fault diagnosis on a number of operating points without having to re-qualifying the networks. Besides, the application of such corrections would allow the use of the measurements degradation, of two or more operating points, which will improve the reliability of the obtained results. The diagnostic process requires to determine the presence of a fault before any further action, such as the fault isolation and his quantification. A nested ANN structure proved useful to fragment activities. This structure would allow easy maintenance of each unit, a rapid requalification in scenarios, where it is required, and finally, a better diagnosis through each specialized network. Numerous tests, applied to the developed case study, demonstrated the high quality of derivable results from ANN. A high degree of accuracy has been obtained with the various processes, which involve classifications, and approximations. Finally, other impacts of this research can be:

- applying the protocol obtained by GT users and GPFD tool manufacturers

- synergistic application of ANN that, thanks to its speed of a solution convergence, can be applied to an isolated failure class. 
In addition, the possible contributions, in authors' opinion, of this research are:

- Development of a parameter-correction approach, that enables the fault diagnosis to be made under different working points, to limit the level of the information accuracy and training that the model used to generate the data;

- Development of an approach for the selection of optimal measure groups, for failures diagnosis. This is particularly useful for scenarios characterized by the data absence or in operating GT plant, where the manager wants to know what are the interesting measurements to improve the observability of particular defects;

- The distinction between a defective and non-defective engine, through measured pattern protocols;

- The sensor fault isolation from component failures, to avoid false alarms, which are characteristic of more traditional diagnostic techniques;

- Evaluation of level defects in single or multiple defective sensors simultaneously;

- The isolation of the defective part(s) at the class level;

- Evaluation of the defect on the isolated failure component(s);

- The introduction of a comprehensive approach involving nested ANN for GPFD.

Finally, the validity of the results obtained in this research has been verified, but for extending the scope of application, the following issues would need to address:

- Measurements have been taken by two or more independent operating points and analyzed without correction, with the advantage of increasing sensory information; this is an approach that can be useful for any engine configuration equipped with few instrumentations;

- The use of parallel processing, to speed up the task of training the network. Some networks typically take several days of setup in scenarios such as this, where most batch networks are required. Using a greater number of processors, a quick setup can be ensured;

- Consideration of problems with a degree of freedom that cause a change of only one operating parameter, for example efficiency or flow rate. As an example, a turbine cooling problem causes a change in the flow rate of the turbine. The proposed model considers performance failures that they used only two degrees of freedom;

- Evaluation of noise and imprecision in the setting of the engine operational parameters;

- The ability to embed GA and ANN, in such a way as to optimize the weight, learning rates, the momentum factor and similar ones, was required by neural networks. This will reduce the ANN training time and restrict the possibility of a sub-optimization using convergence in a local minimum;

- The possibility of fault isolation and evaluation of scenarios with existing failures in sensors and components;

- Consider already online networks trained, i.e., with a continuous updating of the network weights and failures.

\section{References}

Agrawal RK, MacIsaac BD, Saravanamuttoo HIH (1978) An analysis procedure for validation of on-site performance measurements of gas turbines. ASME J Eng Power (78-GT-152)

Aker GF, Saravanamuttoo HIH (1989) Predicting gas turbine performance behavior due to compressor fouling using computer simulation techniques. ASME $\mathrm{J}$ Eng Gas Turbines Power 111:343-350

Anderson D, McNeill G (1992) Artificial neural networks technology. Data and analysis center for software (DACS), Technical Reports. Contract no. F30602-89-C-0082

Angelakis C, Loukis EN, Pouliezos AD, Stavrakakis GS (2001) A neural network based method for gas turbine blading fault diagnosis. Int J Model Simul 21(1):51-60

Bakal B, Adali T, Fakory R, Sonmez MK, Tsaoi O (1995) Neural network simulation of real time core neutronic model. In: Proceedings of the SCS simulation multiconference, Phoenix, AZ, USA

Barlett EB, Uhrig RE (1992) Power plant status diagnostics using artificial neural network. J Nucl Technol 97:272-281

Barschdorff D (1991) Comparison of neural and classical decision algorithms. IFAC/IMACS symposium on fault detection, supervision and safety for technical processes, 10-13 Sept, BadenBaden, Germany

Boyce MP (1984) Gas turbine engineering handbook. Gulf Publishing, Houston

Brotherton T, Jahns G, Jacobs J, Wroblewski D (2000) Prognosis of faults in gas turbine engines. IEEE Aerosp Conf Proc 6:163-171

Brownell TA (1992) Neural networks for sensor management and diagnostics. Aerosp Electron Conf Proc IEEE 3:923-929

Centeno P, Egido I, Domingo C, Fernández F, Rouco L, González M. Review of gas turbine models for power system stability studies. http://aedie.org/9CHLIE-paper-send/368-Centeno.pdf

Cheng B, Titterington DM (1994) Neural networks: a review from a statistical perspective. Stat Sci 9(1):2-54

DePold HR, Gass FD (1999) The application of expert systems and neural networks to gas turbine prognostics and diagnostics. J Eng Gas Turbines Power 121:607-612

Dong DW, Hopfield JJ, Unnikrishnan KP (1997) Neural networks for engine fault diagnostics. Neural Netw Signal Process VII:636-644

ENEA, Department of Energy Technology (DTE), ENEA, Casaccia, Roma. Personal communication (April 2016-July 2016)

Fantoni PF, Mazzola A (1996) Multiple failure signal validation in nuclear power plants using artificial neural networks. Nucl Technol 113(3):368-374

Féraud R, Clérot F (2002) A methodology to explain neural network classification. Neural Netw 15(29):237-246 
Hajagos LM, Bérubé GR (2001) Utility experience with gas turbine testing and modeling. Power Eng Soc Winter Meet IEEE 3. doi:10.1109/PESW.2001.916934
Tamuno-Ojuemi SOT (2003) Advanced gas-path fault diagnostics for stationary gas turbines. PhD thesis, Cranfield University, School of Engineering 